\title{
Mitochondrial Damage: A Target for New Therapeutic Horizons
}

\author{
Jean F. Soustiel* and Sarit Larisch ${ }^{\dagger}$ \\ *Acute Brain Injury Research Laboratory, Faculty of Medicine, Technion-Israel Institute of Technology, 31096 Haifa, Israel; \\ ${ }^{\dagger}$ Cell Death Research Laboratory, Biology Department, Faculty of Sciences, University of Haifa, 31905 Haifa, Israel
}

\begin{abstract}
Summary: Traumatic brain injury (TBI) represents a leading cause of death and morbidity, as well as a considerable social and economical burden in western countries, and has thus emerged as a formidable therapeutic challenge. Yet despite tremendous efforts enlightening the mechanisms of neuronal death, hopes for the "magic bullet" have been repeatedly deceived, and TBI management has remained focused on the control of increased intracranial pressure. Indeed, impairment of cerebral metabolism is traditionally attributed to impaired oxygen delivery mediated by reduced cerebral perfusion in the swollen cerebral parenchyma. Although intuitively appealing, this hypothesis is not entirely supported by physiological facts and does not take into consideration mitochondrial dysfunction that has been repeatedly reported in both human and animal TBI. Although the nature and origin of the events leading to
\end{abstract}

mitochondrial damage may be different, most share a permeabilization of mitochondrial membrane, which therefore may represent a logical target for new therapeutic strategies. Therefore, the proteins mediating these events may represent promising targets for new TBI therapies. Furthermore, mimicking anti-apoptotic proteins, such as Bcl-2 or XIAP, or inhibiting mitochondrial pro-apoptotic proteins, such as Smac/DIABLO, Omi/HTRA2, and ARTS (septin 4 isoform 2) may represent useful novel therapeutic strategies. This review focuses on mechanisms of the mitochondrial membrane permeabilization and its consequences and discusses the current and possible future therapeutic implications of this key event of neuronal death. Key Words: Traumatic brain injury, mitochondria, mitochondrial permeability transition pore, apoptosis, cyclosporin A.

\section{INTRODUCTION}

Traumatic brain injury (TBI) represents a leading cause of death and morbidity, as well as a considerable social and economical burden in western countries. Since the pioneer work of Reilly, ${ }^{1}$ the importance of development of secondary brain damage has gained increasing attention and has been the focus of numerous studies, both in the clinical and experimental settings. Despite extensive experimental research emphasizing the role played by multiple intracellular events, the mainstay of TBI management has remained the control of increased intracranial pressure (ICP). For decades, impairment of cerebral metabolism has been attributed to impaired oxygen delivery, mediated by reduced cerebral perfusion in the swollen cerebral parenchyma. ${ }^{2}$ Accordingly, reduction of ICP is usually argued for restoration of previously compromised cerebral perfusion as a sine qua noncondition for improvement of cerebral metabolism. Although

Address correspondence and reprint requests to: Jean F. Soustiel, Acute Brain Injury Research Laboratory, Faculty of Medicine, Technion-Israel Institute of Technology, Efron St, 1, Haifa 31096, Israel. E-mail: j_soustiel@ rambam.health.gov.il. uncontrolled ICP elevation has been shown to be responsible for reduced oxygen delivery, nonischemic impairment of oxidative metabolism has only recently been recognized as the prominent source of energy crisis triggered by TBI in the presence of cerebral blood flow that is maintained well above the ischemic range in the vast majority of patients. ${ }^{3,4}$ Failure of damaged mitochondria to cope with energy demands is particularly harmful for the neuronal population, considering the high energy demands of neurons that essentially rely on the oxidative metabolism and on the integrity of the electron transport chain.

Accumulating evidence has shown that the mitochondrion has a pivotal role in post traumatic neuronal death by integrating numerous noxious signals responsible for both structural and functional damage on one hand and by amplifying these signals through activation of several proteolytic events leading to cell death. Although the nature and origin of these events may be different, most share permeabilization of the mitochondrial membrane, which therefore may represent a logical target for new therapeutic strategies. The purpose of this article is to review the mechanisms of the mitochondrial membrane 
permeabilization and its consequences, and to discuss the current and possible future therapeutic implications.

\section{MITOCHONDRIAL MEMBRANE PERMEABILIZATION}

A constant observation of both clinical and experimental studies is the impairment of aerobic metabolism developing shortly after TBI. ${ }^{4-6}$ Under normal conditions, oxidative metabolism is assumed by the respiratory complexes of the electron transport chain embedded in the inner mitochondrial membrane. Electron transfer is coupled with pumping of $\mathrm{H}^{+}$across the mitochondrial membrane generating a gradient used by the ATP synthase complex to synthesize the ATP and results in a potential known as transmembrane mitochondrial potential $(\Delta \Psi \mathrm{m})$. After the injury, numerous processes such as $\mathrm{Ca}^{2+}$ accumulation and oxidative stress target the integrity of the mitochondrial membrane, resulting in influx of water and electrolytes with dissipation of the $\Delta \Psi \mathrm{m}$ and loss of mitochondrial functions. This phenomenon, known as mitochondrial membrane permeabilization, may itself follow two distinct pathophysiological pathways involving different molecules within and outside the mitochondria.

\section{Mitochondrial permeability transition}

Across the mitochondrial membrane is a complex of proteins creating a physiological and selective crossing channel between the mitochondrial matrix and the cytosol, known as the mitochondrial permeability transition pore (mPTP) (FIG. 1). Under physiological conditions, the mPTP assumes a low conductance profile, but after brain injury, a forced induction of the MPTP may be triggered by different signals, resulting in a high conductance transition of the MPTP, known as mitochondrial permeability transition. This unselective opening of the mPTP will in turn result in a massive leak of protons and complete and immediate dissipation of the $\Delta \Psi \mathrm{m}$, indicating an irreversible commitment to death. As this phenomenon is complete, outer membrane integrity is compromised, leading to the release of inter-membrane mitochondrial pro-apoptotic proteins, such as cytochrome c, apoptosis inducing factor, and second mitochondria-derived activator of caspase/direct AIP protein with low PI (Smac/DIABLO), all being responsible for activation of caspase-dependent and caspase-independent executioners of apoptosis that are separately discussed. Although the exact molecular structure of the MPTP is still a matter of debate, there is a general consensus on several key components that represent the core of the pore, namely the cyclophilin D (CyPD), the voltage-dependent anion channel (VDAC), and the adenine nucleotide translocase (ANT). ${ }^{7}$

CyPD has been identified as a component of the mPTP embedded in the inner membrane that can be selectively inhibited by cyclosporin A (CsA). ${ }^{8,9}$ Several studies have since then provided a wealthy bulk of evidence showing that the induction of the MPTP correlates with the tissue level of CyPD. ${ }^{10-12}$ In most instances, CyPD deficiency was shown to result in decreased vulnerability to hypoxia, ischemia, or reactive oxygen and nitrogen species mediated injury, ${ }^{11,12}$ all present at least to some degree after severe TBI. More recently, Naga et al. ${ }^{10}$ showed that higher levels of CyPD in post-synaptic mitochondria were associated with a significantly increased vulnerability to mitochondrial permeability transition (MTP). CsA, a well-known immunosuppressive drug, inhibits mPTP induction by binding to the CyPD ${ }^{9,13}$ and presumably cleaving it from the inner membrane. ${ }^{9}$ Prompted by these observations, scientists have investigated the potential therapeutic benefit of CsA in acute brain injury. Indeed, early animal studies provided convincing evidence that administration of CsA results in reduced axonal damage in diffuse axonal injury models ${ }^{14,15}$ and dramatic reduction $(74 \%)$ in the volume of the lesion created by controlled cortical impact. ${ }^{16}$ Noticeably, the authors of both groups were able to show that improved tissue outcome was associated with a protective mitochondrial effect expressed either by preserved functional status ${ }^{17}$ or structural integrity. ${ }^{15}$ Moreover, Alessandri et al. ${ }^{18}$ showed that tissue preservation correlated with improved neurological outcome after experimental TBI. Encouraged by these laboratory data, further studies were performed to provide pharmacological guidelines to further clinical studies ${ }^{19,20}$ and to establish the safety of the drug in respect with its immunosuppressive properties. ${ }^{20,21}$ Based on these data, a prospective randomized and placebo-controlled, double-blinded two-center clinical study was performed in severe human TBI. During the study, patients suffering some severe TBI were enrolled and received $5 \mathrm{mg} / \mathrm{kg}$ during a 24-hour period, delivered within 12 hours from injury. Lactate, pyruvate, and glucose concentrations in the extracellular fluid were measured in samples obtained from a microdialysis catheter as a surrogate for cerebral metabolism. In patients treated with CsA treated, there was a significantly lower lactate/pyruvate ratio, ${ }^{22}$ which is widely admitted to represent an indicator of improved metabolism. ${ }^{23,24} \mathrm{Al}-$ though the impact on clinical outcome was not the primary purpose of this study, numerous studies have established a close correlation between cerebral metabolism and clinical outcome. ${ }^{3,25,26}$ These preliminary results provide the rationale for a larger scale study for investigation of the potential clinical benefit of this appealing drug.

Unlike CyPD, the significance of VDAC in the central nervous system (CNS), apoptosis is much less understood. Although the VDAC seems to represent a key component of the mPTP, its contribution to MTP has not yet been clarified. Several studies have emphasized that 
the variance in the VDAC pore conductance was in inverse proportion with that of the transmembrane potential, suggesting that loss of $\Delta \Psi \mathrm{m}$ could be a potential trigger for opening of the VDAC pore. ${ }^{27,28}$ Conversely, the role of the VDAC in the MTP has been challenged by a recent study showing that VDAC1-, VDAC3-, and VDAC1-/VDAC3-null mice exhibited a $\mathrm{Ca}^{2+}$ and oxidative stress-induced MPT that was similar to that found in wild-type mitochondria, and that wild-type and VDAC-deficient mitochondria and cells exhibited equivalent cytochrome c release, caspase cleavage, and cell death in response to the pro-death Bcl-2 family members Bax and Bid. ${ }^{29,30}$ Eventually, no specific therapy has been designed so far for targeting the VDAC.

Little is known about the ANT and its use for therapeutic targeting seems doubtful in the view of the present knowledge. Although the ANT represents an integral part of the mPTP, ${ }^{31,32}$ its role in MPT remains a matter of controversies. Under physiological conditions, the ANT located at the inner membrane is responsible for an ADP/ATP stoichiometric exchange in which one ADP is imported into the matrix and one ATP is exported into the cytosol. In response to atractyloside, the ANT assumes a different spatial configuration resulting in the creation of a pore. ${ }^{33}$ This rearrangement of the ANT into a lethal pore is inhibited by bongkrekic acid, which selectively binds to the ANT and has proved to reduce cell apoptosis induced by ischemic brain injury. ${ }^{34,35} \mathrm{Al}$ though these observations suggest a therapeutic application targeting the ANT, no pharmacological application has been developed thus far.

\section{Mitochondrial outer membrane permeabilization}

Although the process of MTP eventually results in mitochondrial swelling responsible for a rupture of outer mitochondrial membrane integrity, mitochondrial outer membrane permeabilization (MOMP) does not necessarily imply induction of the MPTP as a prerequisite. As with the MPTP, the outer membrane will integrate death and life signals mostly represented by proteins of the Bcl-2 family. These proteins are characterized by a threedimensional conformation close to that of a channel. ${ }^{36-38}$ These characteristics allow Bax to create channels through lipid membranes large enough to allow the release of cytochrome c. ${ }^{39}$ Under physiological conditions, Bax and Bak exist in a monomeric configuration that does not allow the creation of such channels. However, after activation by truncated Bid, Bax is translocated to the outer membrane where it oligomerizes and assumes a channel-type conformation allowing for release of cytochrome c. ${ }^{40,41}$ Although there is no available therapeutic strategy capable to specifically target this process, modulation of the Bax/Bcl-2 ratio may affect the fate of MOMP, as it has been shown that cells overexpressing $\mathrm{Bcl}-2$ was less vulnerable to the creation of the large pore at the outer membrane, presumably because of reduced presence of Bax at this location. ${ }^{42}$

\section{MODULATORS OF MITOCHONDRIAL MEMBRANE PERMEABILIZATION}

\section{Regulatory mechanisms}

p53. The p53 tumor suppressor is a regulator gene encoding a growth regulatory protein implicated in both cell cycle blocking and cell death through transcriptional programs. However, in its cytosolic location, p53 has been shown to promote mitochondrial apoptosis by transcription-independent pathways interacting with pro-apoptotic Bcl family members. ${ }^{43}$ Furthermore, p53 mitochondrial translocation has proved to correlate with enhanced neuronal death, ${ }^{44}$ whereas p53 null mice were found to be less vulnerable to ischemia ${ }^{45}$ in comparison with wild type animals. These observations have raised interest regarding the possible therapeutic implications of p53 inhibition, using a specific inhibiting drug, pifithrin- $\alpha$ (PFT- $\alpha)$. Several reports have provided accumulating evidence that PFT- $\alpha$ is neuroprotective, enhancing neuronal survival after amyloid $\beta$-peptide mediated injury ${ }^{46,47}$ or in various models of ischemic brain injury. ${ }^{46,48}$ Furthermore, in a recent study, Luo et al. ${ }^{49}$ found that improved functional recovery from ischemic stroke in rats treated by PFT- $\alpha$ in a delayed fashion correlated with enhanced survival of endogenous progenitor cells in the subventricular zone in a dose-dependent fashion.

Bcl-2 family. The Bcl-2 proteins family includes pro- and anti-apoptotic members that exercise a major regulatory function over the process of cell death. The delicate balance between the expression of the opposing components of the family and their respective impact over the fate of apoptosis has been illustrated as a rheostat by Korsmeyer at al. ${ }^{50}$ Several reports have suggested that the VDAC may represent the molecular basis for the Bax/Bcl-2 rheostat. ${ }^{51,52}$ In this scenario, described by Tsujimoto and Shimizu, ${ }^{51}$ MTP would be the consequence of interaction between the VDAC and proteins of the $\mathrm{Bcl}$ family where $\mathrm{Bcl}-2$ and $\mathrm{Bcl}_{-\mathrm{XL}}$ inhibit MPT and $\mathrm{Bax}$ and Bid promote the opening of the pore. ${ }^{52} \mathrm{~A}$ similar competitive mechanism has been described at the level of the ANT, whose activation is regulated by Bax that enhances the pore formation and $\mathrm{Bcl}-2$ that inhibits it. $^{53,54}$

Although there is no available therapeutic option capable to specifically target the $\mathrm{Bax} / \mathrm{Bcl}-2$, hyperbaric oxygen therapy has been shown to reduce apoptosis ${ }^{55}$ through a mechanism involving overexpression of Bcl-2 and $\mathrm{Bcl}^{-}{ }_{\mathrm{XL}}$ in the perilesional area. ${ }^{30}$ Noticeably, animal studies have shown that this anti-apoptotic effect was associated with partial restoration of $\Delta \Psi \mathrm{m}^{56}$ and improvement of oxidative metabolism. ${ }^{6,57}$ These experi- 
mental findings were later supported by the results of a prospective, cohort-matched microdialysis study performed in 52 patients suffering from severe TBI. ${ }^{58}$ In this study, normobaric hyperoxia was delivered during a 24-h period, starting 6-h post-injury, which resulted in increased glucose and decreased glutamate and lactate levels in treated patients that correlated with a significantly better outcome.

Another route of neuroprotection involving Bcl-2 expression is represented by estrogens. Numerous studies of ischemic and TBI have provided a large bulk of evidence supporting the neuroprotective effect of estrogens. Although various hypotheses have been proposed to explain the neuroprotective effect of estrogens in brain injury such as augmentation of cerebral blood flow ${ }^{59}$ usually linked to the increase of nitric oxide availability, ${ }^{60}$ anti-oxidant activity, ${ }^{61}$ and reduction of excitotoxic effects mediated by glutamate release, ${ }^{62}$ accumulating evidence links estrogens to the bcl-2 family of proteins. In ischemic brain injury, bcl-2 mRNA expression has been found to be higher in the penumbra area in female and estradiol-treated male rats than in estrogen deficient animals. ${ }^{63}$ Similarly, Dubal et al. ${ }^{64}$ found that bcl-2 mRNA was increased in the ischemic cortex of estradioltreated animals. These observations have been supported by similar findings obtained with different types of TBI, suggesting that enhanced bcl-2 expression may account for at least part of the neuroprotection mediated by estrogens. ${ }^{65,66}$ Yet, enthusiasm risen by these encouraging data has somehow been dampened by recent clinical evidence showing that women may have worse outcomes than men. ${ }^{67}$

$18 \mathrm{kDa}$ translocator protein. The $18 \mathrm{kDa}$ translocator protein (TSPO), also known as peripheral benzodiazepine receptor, is a binding protein complex, distinct from the central benzodiazepine receptor that is located at the outer mitochondrial membrane. ${ }^{68}$ Although TSPOs are not usually considered as a part of the MPTP, per se, but they are closely located to the pore and intimately related to the $32-\mathrm{kDa}$ VDAC and to the $30-\mathrm{kDa}$ ANT, ${ }^{69}$ suggesting that it may be involved with the control of mPTP and the mitochondrial pathway of apoptosis. ${ }^{70}$ Supporting this assumption, numerous studies have provided increasing evidence that modulation of TSPO activity by various specific ligands could significantly affect the apoptotic process. ${ }^{71,72}$ Based on this hypothesis, Soustiel et al. ${ }^{73}$ showed that intraperitoneal administration of Ro5-4864 (a TSPO ligand) to rats submitted to cortical contusion, resulted in enhanced neuronal survival, axonal preservation, and reduced activation of caspases 3 and 9. Furthermore, incubation of isolated mitochondria in the presence of Ro5-4864, in addition to Bax, could partially restore the loss of $\Delta \Psi \mathrm{m}$ created by Bax alone. Although Ro5-4864 does not represent a therapeutic option in human TBI because of its epileptogenic properties, the TSPO in the near future may represent an attractive target for the development of new molecules.

\section{DOWNSTREAM EXECUTIONERS}

Although blocking of mitochondrial membrane permeabilization (MMP) represents a rational and potentially efficient means of neuroprotection, design of new therapeutic strategies should take into account the fact that treatments for TBIs can be initiated hours after injury at best so that some degree of MMP should be expected. Consequently, inhibition of post-mitochondrial downstream executioners represents an important and inseparable target that may theoretically result in significant neuroprotection.

\section{Caspases}

The main executioners of apoptosis are caspases, a family of proteases harboring a cysteine residue at their active site that preferentially cleave substrates after aspartate. ${ }^{74-76}$ Caspase family members are divided into two main categories, the initiator caspases, which are usually activated first by apoptotic signals, and the effector caspases. ${ }^{74-76}$ Initiator caspases (caspase-2, 8, 9, and 10) exist as monomers and are activated through binding to adaptor molecules, which enable their oligomerization and activation. These activated initiator caspases can now cleave inactive forms of effector caspases, which amplify the death signaling cascade to terminate the process. Effector caspases (e.g., caspase-3, 6 , and 7) cleave a large range of cytoplasmic and nuclear substrates ultimately leading to irreversible death of the cell. ${ }^{74,76-78}$ Within the mitochondrial pathway, initiator caspase 9 is activated through the formation of the socalled "apoptosome." The apoptosome is assembled when seven heterodimers of apoptotic protease activating factor-1 (Apaf-1), join together with cytochrome c released from mitochondria after MMP to form a symmetrical "wheel" that binds to caspase- 9 and promotes its activation. ${ }^{79,80}$ Cleavage of caspase- 9 , in turn, results in activation of effector caspase-3, which appears to play a crucial role in brain apoptosis. ${ }^{81}$ Consequently, inhibition or reduction of caspases activity has been considered as a possible avenue of neuroprotection.

Several studies using nonspecific caspase inhibitors, such as Z-VAD-fmk (pan-caspase inhibitor), have reported a neuroprotective effect in animal models of TBI. ${ }^{82,83}$ Selective reversible inhibitors of caspase- $3^{84,85}$ and caspase $-9^{86}$ have also proved to promote neuronal survival after hypoxic ischemic injury in the cortex of newborn rats. Importantly, neuroprotection can still be achieved when intracerebral administration of $\operatorname{DEVD}(\mathrm{OMe})-\mathrm{CH} 2 \mathrm{~F}$ is delayed by 6 to $9 \mathrm{~h}$ after mild transient focal ischemia ${ }^{87}$ or cerebral hypoxia. ${ }^{88}$ This prolonged therapeutic window makes caspase inhibitors 
particularly attractive for the treatment of stroke. Molecular targeting of the Apaf-1/caspase-9 pathway as a neuroprotective strategy has been recently reported by Cao et al. $^{89}$ These authors describe the cloning of a novel Apaf-1-interacting protein (AIP) from rat brain. AIP is a short variant of caspase- 9 , which contains the caspaserecruiting domain, but lacks the catalytic domains that caspase-9 possesses. Using an adeno-associated virus vector carrying the AIP cDNA by intracerebral injection, Cao et al. ${ }^{89}$ were able to show a robust AIP expression in the hippocampus that significantly correlated with enhanced CA1 neuronal survival after transient global cerebral ischemia. More recently, a chemical inhibitor of Apaf-1 has been shown to exert cytoprotective effects in vitro by limiting MOMP and caspase- 9 activation. ${ }^{90}$

Despite these encouraging experimental data, the survival efficacy of caspase inhibition in vivo has several caveats and limitations that await further elucidatory studies. For example, selective caspase- 3 inhibition has proved to confer limited anti-apoptotic protection in both ischemic $^{91}$ and traumatic ${ }^{92}$ brain injury, suggesting that inhibition of other apoptosis-inducing elements might be important for a successful rescue of cells after an ischemic insult. ${ }^{93}$ In addition, the efficiency of z-VAD may vary from newborn to adult animals in conferring neuroprotection against ischemia. $^{94}$

\section{Inhibitors of apoptosis proteins: the natural caspase inhibitors}

Expectedly, the apoptotic process is physiologically controlled by both activators and inhibitors of caspases that may represent additional potential targets for neuroprotection (FIG. 2). The best studied caspase inhibitors are the inhibitors of apoptosis proteins (IAPs). ${ }^{95}$ Thus far, eight IAP proteins have been identified in mammals: neuronal AIP, cIAP1, cIAP2, X-linked IAP (XIAP), melanoma IAP, IAP-like protein 2, survivin, and BRUCE/Apollon. ${ }^{95}$ In vitro studies have identified endogenous XIAP as a critical regulator of neuronal apoptosis, ${ }^{96}$ and data from studies of mice overexpressing XIAP have shown that increased levels of this protein confer protection against hypoxia-ischemia-induced caspase activation in both the adult ${ }^{97}$ and neonatal brain..$^{98}$ In a model of transient cerebral ischemia in rats, XIAP overexpression increased cell survival by 5 to 6-fold and improved functional recovery. ${ }^{99}$

Pharmacological implementation of XIAP-based neurorescuing has been successfully reported by Fan et al. ${ }^{100}$ in a model of transient brain ischemia. In this study, intraperitoneal injection of XIAP-derived chimeric polypeptides that included a membrane translocation domain that resulted in limited caspase activation and DNA fragmentation, and improved neurological outcomes after transient ischemia. Thus, developing small molecules that selectively upregulate XIAP may therefore have therapeutic potential for the treatment of both ischemic and traumatic brain injury. The observation that XIAP deficient mice do not exhibit any increased apoptosis in their brain supports this approach. ${ }^{101}$

\section{Mitochondrial IAP-antagonists}

This inhibition of caspases activity by IAPs is balanced by mitochondrial proteins released during the MMP process and acting as IAP antagonists that may be considered as potential therapeutic targets. Three mitochondrial proteins have been identified in mammalian cells: Smac/DIABLO, ${ }^{102,103}$ Omi/ Htra2 ${ }^{104}$ and ARTS ${ }^{105,106}$ (apoptosis-related protein in TGF- $\beta$ signaling pathway). All three proteins have been shown to inhibit IAPs by physical sequestration, ${ }^{104,105,107}$ although ARTS and Omi/Htra2 can specifically target XIAP and reduce its levels. ${ }^{105,108}$ The potential therapeutic implications of IAP antagonist targeting has been recently illustrated by $\mathrm{Su}$ et al. ${ }^{109}$ in a model of ischemia-reperfusion brain injury in rats. Intraperitoneal injection of a novel Omi/HtrA2 inhibitor, UCF-101, $30 \mathrm{~min}$ prior to reperfusion and after $2 \mathrm{~h}$ of middle cerebral artery occlusion, resulted in a significant

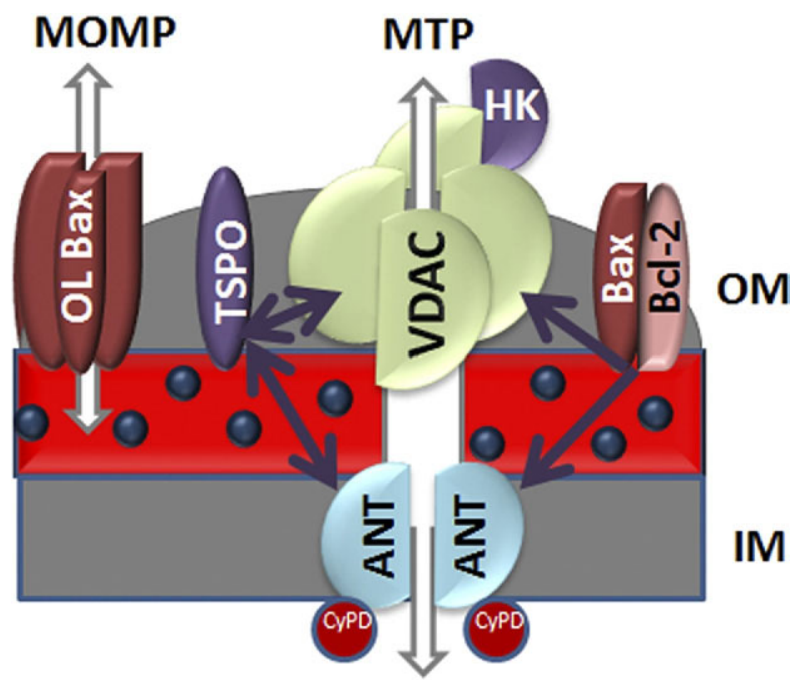

FIG. 1. Mitochondrial permeability transition pore (mPTP) schematic representation of MMP may develop according two different scenarios: mitochondrial permeability transition (MPT) or mitochondrial outer membrane permeabilization (MOMP). During mitochondrial permeability transition (MTP), mitochondrial damage caused by oxidative stress or calcium overload initiates induction of the MPTP at the inner membrane. Opening of this large complex channel results in leaking of protons and massive entry of water and solutes with subsequent swelling of the mitochondria, dissipation of transmembrane mitochondrial potential $(\Delta \Psi \mathrm{m})$, failure of oxidative metabolism and eventually breakage of the outer mitochondrial membrane. This process is modulated by $\mathrm{Bcl}-2$ family proteins and TSPO both interacting with the voltage-dependent anion channel (VDAC) and the adenine nucleotide translocator (ANT). On the other hand, MOMP may develop by translocation and oligomerization of Bax that creates small channels across the OM allowing for release of cytochrome c (dark grey dots). CyPD = cyclophilin D; HK = hexokinase; IM = inner mitochondrial membrane; OL Bax = oligomerized Bax; $\mathrm{OM}=$ outer mitochondrial membrane; $\mathrm{TSPO}=18 \mathrm{kDa}$ translocator protein formerly known as peripheral benzodiazepine receptor. 


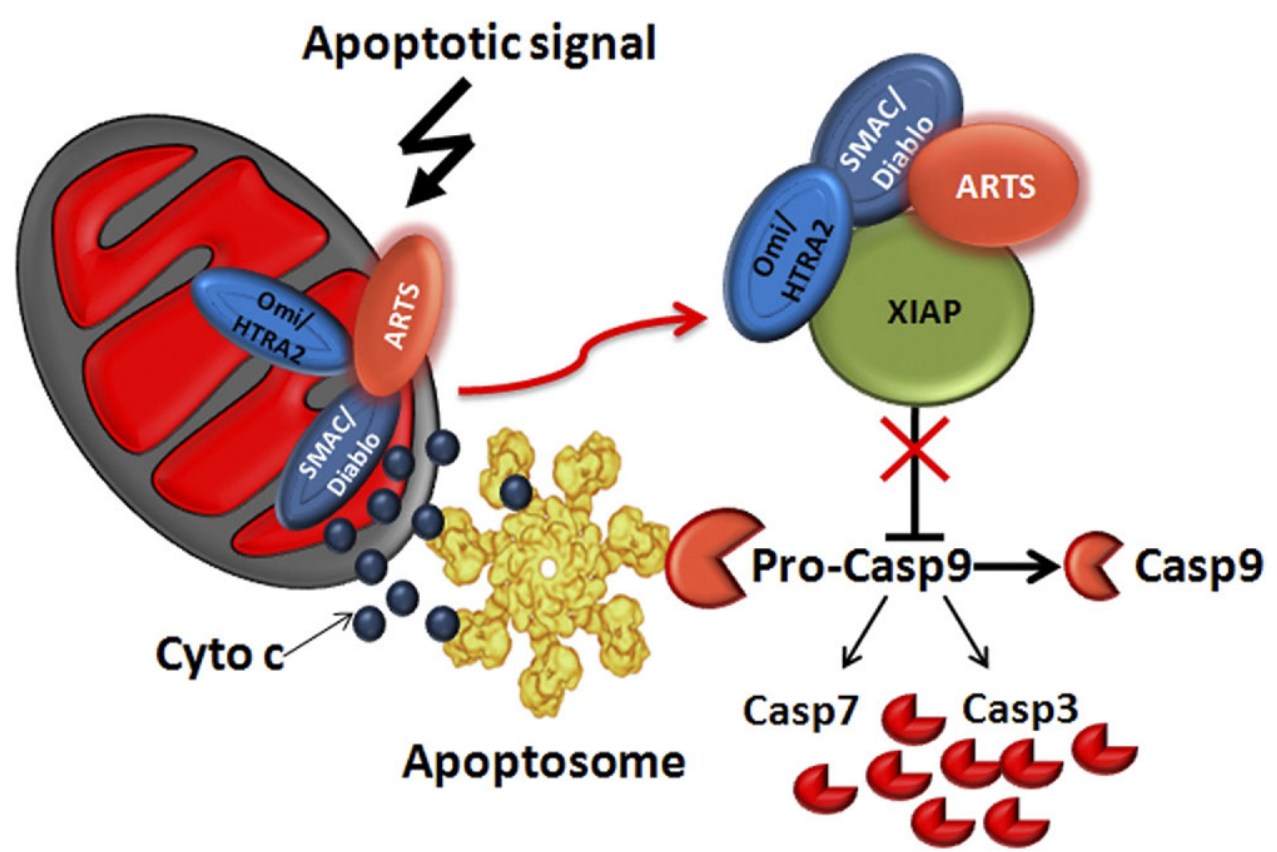

FIG. 2. Downstream post-mitochondrial executioners and their modulators MMP results in cytochrome c (cyto c; dark grey dots) release in the cytosol, contributing with Apaf-1 to the assembly of the apoptosome that activates Caspase-9 (Casp-9), initiating the caspase pathway of cell death through activation of effectors Casp-3 and Casp-7. This process is negatively regulated by the inhibitors of apoptosis proteins (IAPS) mostly represented by the X-linked IAP (XIAP). The XIAP, in turn, is deactivated by SMAC/Diablo, Omi/HTRA2, and ARTS.

reduction of TUNEL positive cells that correlated with concurrent reduction in caspase-3 activity.

Another mitochondrial protein that promotes apoptosis through binding and antagonizing XIAP is ARTS..$^{105,106}$ Although, so far, most efforts have been focused on Smac/DIABLO and Omi/HtrA2, ARTS appears to act earlier in the apoptotic cascade and directly inhibits XIAP by a distinct mechanism. ${ }^{105}$ ARTS (septin 4 isoform 2, Genebank accession number gi 17986245 ref NP_536340.1) is derived by differential splicing from the human septin gene Sept4. ${ }^{106}$ ARTS is exceptional, both in terms of its mitochondrial localization and its pro-apoptotic function, not shared by any other known human septin. Several lines of evidence suggest that ARTS induces apoptosis through XIAP inhibition in brain injury. First, ARTS was shown to be highly expressed in the brain ${ }^{106}$ where septins generally play a significant role in various neuropathologies. Second, ARTS was found to function as a potent IAP-antagonist both in vitro ${ }^{105}$ and in vivo. ${ }^{110}$ Third, under apoptotic conditions ARTS is released from mitochondria, directly binds to XIAP in the cytosol, reducing its levels through degradation via the ubiquitin-proteosome system. ${ }^{105}$ Fourth, several studies implicate XIAP nuclear translocation in neuronal death and suggest that this translocation may be a novel mechanism regulating hypoxic-ischemic brain injury. ${ }^{111}$ ARTS can bind XIAP in the cytosol and translocate in a complex with XIAP to the nucleus. Moreover, a significant increase in nuclear localization of XIAP was shown when XIAP was co- overexpressed with ARTS, as compared to XIAP alone. ${ }^{105}$ Taken together, these observations suggest that ARTS plays an important role in promoting apoptosis through XIAP inhibition in the brain and may therefore represent an additional potential target for design of new therapeutic agents (FIG. 2).

\section{Apoptosis-inducing factor}

Among the inter-membrane proteins released by MMP, the role of apoptosis-inducing factor in generation of secondary neuronal death in TBI has not yet been fully elucidated. Several preliminary studies using various models of ischemic brain injury have suggested that release of AIF by the MMP process could represent the first step of caspase-independent apoptosis. ${ }^{112,113}$ In a recent animal study, Slemmer et al. ${ }^{114}$ have shown in an in vitro model of axonal injury that AIF translocated to the nucleus and was responsible for chromatine condensation independently from caspase activity. Furthermore, the authors showed that down-regulation of AIF in vitro by small inhibitory RNA resulted in a significant attenuation of neuronal cell death by $67 \%$ induced by stretching. This observation was further supported in vivo by a similar reduction in contusion expansion in AIF-deficient harlequin mutant mice. To date, however, no therapeutic strategy specifically targeting the AIF has been investigated yet, so that eventually prevention or reduction of MMP remains the only available way to affect AIFmediated damage. 


\section{CONCLUDING REMARKS}

Considerable laboratory data have emphasized the crucial impact of mitochondrial membrane permeabilization and its consequences over the fate of damaged neurons, enhancing the potential therapeutic implications of this pivotal phenomenon. Although most of these data are still preliminary or even merely speculative in nature, few therapeutic agents, such as cyclosporin A with robust evidence of their neuroprotective effect can be easily and readily implemented without interfering with standard management and can not be ignored any further. In the face of failure of our current care measures to prevent or reverse mitochondrial damage, time has come to move from a simplistic biomechanical approach limited to control of intracranial pressure to a more pathophysiological strategy and to call for large scale multicenter studies.

\section{REFERENCES}

1. Reilly PL, Graham DI, Adams JH, et al. Patients with head injury who talk and die. Lancet 1975;2:375-377.

2. Rosner MJ, Rosner SD, Johnson AH. Cerebral perfusion pressure: management protocol and clinical results. J Neurosurg 1995;83:949-962.

3. Soustiel JF, Sviri GE. Monitoring of cerebral metabolism: nonischemic impairment of oxidative metabolism following severe traumatic brain injury. Neurol Res 2007;29:654-660.

4. Vespa P, Bergsneider M, Hattori N, et al. Metabolic crisis without brain ischemia is common after traumatic brain injury: a combined microdialysis and positron emission tomography study. J Cereb Blood Flow Metab 2005;25:763-774.

5. Soustiel JF, Glenn TC, Shik V, et al. Monitoring of cerebral blood flow and metabolism in traumatic brain injury. J Neurotrauma 2005;22:955-965.

6. Zhou Z, Daugherty WP, Sun D, et al. Protection of mitochondrial function and improvement in cognitive recovery in rats treated with hyperbaric oxygen following lateral fluid-percussion injury. J Neurosurg 2007;106:687-694.

7. Galluzzi L, Blomgren K, Kroemer G. Mitochondrial membrane permeabilization in neuronal injury. Nat Rev Neurosci 2009;10: 481-494.

8. Bernardi P, Broekemeier KM, Pfeiffer DR. Recent progress on regulation of the mitochondrial permeability transition pore; a cyclosporin-sensitive pore in the inner mitochondrial membrane. J Bioenerg Biomembr 1994;26:509-517.

9. Szabo I, Zoratti M. The giant channel of the inner mitochondrial membrane is inhibited by cyclosporin A. J Biol Chem 1991;266: 3376-3379.

10. Naga KK, Sullivan PG, Geddes JW. High cyclophilin D content of synaptic mitochondria results in increased vulnerability to permeability transition. J Neurosci 2007;27:7469-7475.

11. Schinzel AC, Takeuchi O, Huang Z, et al. Cyclophilin D is a component of mitochondrial permeability transition and mediates neuronal cell death after focal cerebral ischemia. Proc Natl Acad Sci USA 2005;102:12005-12010.

12. Wang X, Carlsson Y, Basso E, et al. Developmental shift of cyclophilin D contribution to hypoxic-ischemic brain injury. J Neurosci 2009;29:2588-2596.

13. Broekemeier KM, Pfeiffer DR. Inhibition of the mitochondrial permeability transition by cyclosporin A during long time frame experiments: relationship between pore opening and the activity of mitochondrial phospholipases. Biochemistry 1995;34:1644016449.

14. Okonkwo DO, Buki A, Siman R, et al. Cyclosporin A limits calcium-induced axonal damage following traumatic brain injury. Neuroreport 1999;10:353-358.
15. Okonkwo DO, Povlishock JT. An intrathecal bolus of cyclosporin A before injury preserves mitochondrial integrity and attenuates axonal disruption in traumatic brain injury. J Cereb Blood Flow Metab 1999;19:443-451.

16. Sullivan PG, Thompson M, Scheff SW. Continuous infusion of cyclosporin A postinjury significantly ameliorates cortical damage following traumatic brain injury. Exp Neurol 2000;161:631637.

17. Sullivan PG, Thompson MB, Scheff SW. Cyclosporin A attenuates acute mitochondrial dysfunction following traumatic brain injury. Exp Neurol 1999;160:226-234.

18. Alessandri B, Rice AC, Levasseur J, et al. Cyclosporin A improves brain tissue oxygen consumption and learning/memory performance after lateral fluid percussion injury in rats. J Neurotrauma 2002;19:829-841.

19. Empey PE, McNamara PJ, Young B, et al. Cyclosporin A disposition following acute traumatic brain injury. J Neurotrauma 2006;23:109-116.

20. Hatton J, Rosbolt B, Empey P, et al. Dosing and safety of cyclosporine in patients with severe brain injury. J Neurosurg 2008; 109:699-707.

21. Mazzeo AT, Kunene NK, Gilman CB, et al. Severe human traumatic brain injury, but not cyclosporin a treatment, depresses activated T lymphocytes early after injury. J Neurotrauma 2006; 23:962-975.

22. Mazzeo AT, Alves OL, Gilman CB, et al. Brain metabolic and hemodynamic effects of cyclosporin A after human severe traumatic brain injury: a microdialysis study. Acta Neurochir (Wien) 1008;150:1019-1031.

23. Marion DW, Puccio A, Wisniewski SR, et al. Effect of hyperventilation on extracellular concentrations of glutamate, lactate, pyruvate, and local cerebral blood flow in patients with severe traumatic brain injury. Crit Care Med 2002;30:2619-2625.

24. Vespa P, Bergsneider M, Hattori N, et al. Metabolic crisis without brain ischemia is common after traumatic brain injury: a combined microdialysis and positron emission tomography study. J Cereb Blood Flow Metab 2005;25:763-774.

25. Le Roux PD, Newell DW, Lam AM, et al. Cerebral arteriovenous oxygen difference: a predictor of cerebral infarction and outcome in patients with severe head injury. J Neurosurg 1997;87:1-8.

26. Marcoux J, McArthur DA, Miller C, et al. Persistent metabolic crisis as measured by elevated cerebral microdialysis lactatepyruvate ratio predicts chronic frontal lobe brain atrophy after traumatic brain injury. Crit Care Med 2008;36:2871-2877.

27. Benz R. Permeation of hydrophilic solutes through mitochondrial outer membranes: review on mitochondrial porins. Biochim Biophys Acta 1994;1197:167-196.

28. Colombini M. Voltage gating in the mitochondrial channel, VDAC. J Membr Biol 1989;111:103-111.

29. Baines CP, Kaiser RA, Sheiko T, et al. Voltage-dependent anion channels are dispensable for mitochondrial-dependent cell death. Nat Cell Biol 2007;9:550-555.

30. Vlodavsky E, Palzur E, Feinsod M, et al. Evaluation of the apoptosis-related proteins of the BCL-2 family in the traumatic penumbra area of the rat model of cerebral contusion, treated by hyperbaric oxygen therapy: a quantitative immunohistochemical study. Acta Neuropathol 2005;110:120-126.

31. Belzacq AS, Vieira HL, Kroemer G, et al. The adenine nucleotide translocator in apoptosis. Biochimie 2002;84:167-176.

32. Le Quoc K, Le Quoc D. Involvement of the ADP/ATP carrier in calcium-induced perturbations of the mitochondrial inner membrane permeability: importance of the orientation of the nucleotide binding site. Arch Biochem Biophys 1988;265:249-257.

33. Brandolin G, Dupont Y, Vignais PV. Substrate-induced modifications of the intrinsic fluorescence of the isolated adenine nucleotide carrier protein: demonstration of distinct conformational states. Biochemistry 1985;24:1991-1997.

34. Cao G, Minami M, Pei W, et al. Intracellular Bax translocation after transient cerebral ischemia: implications for a role of the mitochondrial apoptotic signaling pathway in ischemic neuronal death. J Cereb Blood Flow Metab 2001;21:321-333. 
35. Muranyi M, Li PA. Bongkrekic acid ameliorates ischemic neuronal death in the cortex by preventing cytochrome $\mathrm{c}$ release and inhibiting astrocyte activation. Neurosci Lett 2005;384:277-281.

36. McDonnell JM, Fushman D, Milliman CL, et al. Solution structure of the proapoptotic molecule BID: a structural basis for apoptotic agonists and antagonists. Cell 1999;96:625-634.

37. Petros AM, Medek A, Nettesheim DG, et al. Solution structure of the antiapoptotic protein bcl-2. Proc Natl Acad Sci USA 2001; 98:3012-3017.

38. Suzuki M, Youle RJ, Tjandra N. Structure of Bax: coregulation of dimer formation and intracellular localization. Cell 2000;103: 645-654.

39. Saito M, Korsmeyer SJ, Schlesinger PH. BAX-dependent transport of cytochrome c reconstituted in pure liposomes. Nat Cell Biol 2000;2:553-555.

40. Antonsson B, Montessuit S, Lauper S, et al. Bax oligomerization is required for channel-forming activity in liposomes and to trigger cytochrome c release from mitochondria. Biochem J 2000; 345(pt 2):271-278.

41. Desagher S, Osen-Sand A, Nichols A, et al. Bid-induced conformational change of Bax is responsible for mitochondrial cytochrome c release during apoptosis. J Cell Biol 1999;144:891-901.

42. Pavlov EV, Priault M, Pietkiewicz D, et al. A novel, high conductance channel of mitochondria linked to apoptosis in mammalian cells and Bax expression in yeast. J Cell Biol 2001;155: 725-731.

43. Moll UM, Wolff S, Speidel D, et al. Transcription-independent pro-apoptotic functions of p53. Curr Opin Cell Biol 2005; 17:631636.

44. Endo H, Kamada H, Nito C, et al. Mitochondrial translocation of p53 mediates release of cytochrome c and hippocampal CA1 neuronal death after transient global cerebral ischemia in rats. J Neurosci 2006;26:7974-7983.

45. Yonekura I, Takai K, Asai A, et al. p53 potentiates hippocampal neuronal death caused by global ischemia. J Cereb Blood Flow Metab 2006;26:1332-1340.

46. Culmsee C, Zhu X, Yu QS, et al. A synthetic inhibitor of $\mathrm{p} 53$ protects neurons against death induced by ischemic and excitotoxic insults, and amyloid beta-peptide. J Neurochem 2001;77: $220-228$.

47. Strosznajder RP, Jesko H, Banasik M, et al. Effects of p53 inhibitor on survival and death of cells subjected to oxidative stress. J Physiol Pharmacol 2005;56(suppl 4):215-221.

48. Leker RR, Aharonowiz M, Greig NH, et al. The role of p53induced apoptosis in cerebral ischemia: effects of the p53 inhibitor pifithrin alpha. Exp Neurol 2004;187:478-486.

49. Luo Y, Kuo CC, Shen H, et al. Delayed treatment with a p53 inhibitor enhances recovery in stroke brain. Ann Neurol 2009;65: $520-530$.

50. Korsmeyer SJ, Shutter JR, Veis DJ, et al. Bcl-2/Bax: a rheostat that regulates an anti-oxidant pathway and cell death. Semin Cancer Biol 1993;4:327-332.

51. Shimizu S, Narita M, Tsujimoto Y. Bcl-2 family proteins regulate the release of apoptogenic cytochrome c by the mitochondrial channel VDAC. Nature 1999;399:483-487.

52. Tsujimoto Y, Shimizu S. VDAC regulation by the Bcl-2 family of proteins. Cell Death Differ 2000;7:1174-1181.

53. Brenner C, Cadiou H, Vieira HL, et al. Bcl-2 and Bax regulate the channel activity of the mitochondrial adenine nucleotide translocator. Oncogene 2000;19:329-336.

54. Marzo I, Brenner C, Zamzami N, et al. The permeability transition pore complex: a target for apoptosis regulation by caspases and bcl-2-related proteins. J Exp Med 1998;187:1261-1271.

55. Palzur E, Vlodavsky E, Mulla H, et al. Hyperbaric oxygen therapy for reduction of secondary brain damage in head injury: an animal model of brain contusion. J Neurotrauma 2004;21:41-48.

56. Palzur E, Zaaroor M, Vlodavsky E, et al. Neuroprotective effect of hyperbaric oxygen therapy in brain injury is mediated by preservation of mitochondrial membrane properties. Brain Res 2008;1221:126-133.

57. Daugherty WP, Levasseur JE, Sun D, et al. Effects of hyperbaric oxygen therapy on cerebral oxygenation and mitochondrial func- tion following moderate lateral fluid-percussion injury in rats. J.Neurosurg 2004;101:499-504.

58. Tolias CM, Reinert M, Seiler R, et al. Normobaric hyperoxiainduced improvement in cerebral metabolism and reduction in intracranial pressure in patients with severe head injury: a prospective historical cohort-matched study. J Neurosurg 2004;101: $435-444$.

59. Roof RL, Hall ED. Estrogen-related gender difference in survival rate and cortical blood flow after impact-acceleration head injury in rats. J Neurotrauma 2000;17:1155-1169.

60. Geary GG, Krause DN, Duckles SP. Estrogen reduces mouse cerebral artery tone through endothelial NOS- and cyclooxygenase-dependent mechanisms. Am J Physiol Heart Circ Physiol 2000;279:H511-H519.

61. Tang M, Abplanalp W, Ayres S, et al. Superior and distinct antioxidant effects of selected estrogen metabolites on lipid peroxidation. Metabolism 1996;45:411-414.

62. Zaulyanov LL, Green PS, Simpkins JW. Glutamate receptor requirement for neuronal death from anoxia-reoxygenation: an in Vitro model for assessment of the neuroprotective effects of estrogens. Cell Mol Neurobiol 1999;19:705-718.

63. Alkayed NJ, Goto S, Sugo N, et al. Estrogen and Bcl-2: gene induction and effect of transgene in experimental stroke. J Neurosci 2001;21:7543-7550.

64. Dubal DB, Shughrue PJ, Wilson ME, et al. Estradiol modulates bcl-2 in cerebral ischemia: a potential role for estrogen receptors. J Neurosci 1999;19:6385-6393.

65. Soustiel JF, Palzur E, Nevo O, et al. Neuroprotective anti-apoptosis effect of estrogens in traumatic brain injury. J Neurotrauma 2005;22:345-352.

66. Wise PM, Dubal DB, Wilson ME, et al. Estradiol is a neuroprotective factor in in vivo and in vitro models of brain injury. J Neurocytol 2000;29:401-410.

67. Farace E, Alves WM. Do women fare worse: a metaanalysis of gender differences in traumatic brain injury outcome. J Neurosurg 2000;93:539-545.

68. Braestrup C, Squires RF. Specific benzodiazepine receptors in rat brain characterized by high-affinity $(3 \mathrm{H})$ diazepam binding. Proc Natl Acad Sci USA 1977;74:3805-3809.

69. McEnery MW, Snowman AM, Trifiletti RR, et al. Isolation of the mitochondrial benzodiazepine receptor: association with the voltage-dependent anion channel and the adenine nucleotide carrier. Proc Natl Acad Sci USA 1992;89:3170-3174.

70. Decaudin D. Peripheral benzodiazepine receptor and its clinical targeting. Anticancer Drugs 2004;15:737-745.

71. Bono F, Lamarche I, Prabonnaud V, et al. Peripheral benzodiazepine receptor agonists exhibit potent antiapoptotic activities. Biochem Biophys Res Commun 1999;265:457-461.

72. Decaudin D, Castedo M, Nemati F, et al. Peripheral benzodiazepine receptor ligands reverse apoptosis resistance of cancer cells in vitro and in vivo. Cancer Res 2002;62:1388-1393.

73. Soustiel JF, Zaaroor M, Vlodavsky E, et al. Neuroprotective effect of Ro5-4864 following brain injury. Exp Neurol 2008; 214:201-208.

74. Boyce M, Degterev A, Yuan J. Caspases: an ancient cellular sword of Damocles. Cell Death Differ 2004;11:29-37.

75. Nicholson DW. Caspase structure, proteolytic substrates, and function during apoptotic cell death. Cell Death Differ 1999;6: $1028-1042$.

76. Thornberry NA, Lazebnik Y. Caspases: enemies within. Science 1998;281:1312-1316.

77. Muzio M, Salvesen GS, Dixit VM. FLICE induced apoptosis in a cell-free system. Cleavage of caspase zymogens. J Biol Chem 1997;272:2952-2956.

78. Yuan J, Shaham S, Ledoux S, et al. The C. elegans cell death gene ced-3 encodes a protein similar to mammalian interleukin-1 betaconverting enzyme. Cell 1993;75:641-652.

79. Rodriguez J, Lazebnik Y. Caspase-9 and APAF-1 form an active holoenzyme. Genes Dev 1999;13:3179-3184.

80. Srinivasula SM, Ahmad M, Fernandes-Alnemri T, et al. Autoactivation of procaspase-9 by Apaf-1-mediated oligomerization. Mol Cell 1998;1:949-957. 
81. Kuida K, Zheng TS, Na S, et al. Decreased apoptosis in the brain and premature lethality in CPP32-deficient mice. Nature 1996; 384:368-372.

82. Clark RS, Nathaniel PD, Zhang X, et al. boc-Aspartyl(OMe)fluoromethylketone attenuates mitochondrial release of cytochrome $\mathrm{c}$ and delays brain tissue loss after traumatic brain injury in rats. J Cereb Blood Flow Metab 2007;27:316-326.

83. Li M, Ona VO, Chen M, et al. Functional role and therapeutic implications of neuronal caspase- 1 and -3 in a mouse model of traumatic spinal cord injury. Neuroscience 2000;99:333-342.

84. Han BH, Xu D, Choi J, et al. Selective, reversible caspase-3 inhibitor is neuroprotective and reveals distinct pathways of cell death after neonatal hypoxic-ischemic brain injury. J Biol Chem 2002;277:30128-30136.

85. Yakovlev AG, Knoblach SM, Fan L, et al. Activation of CPP32like caspases contributes to neuronal apoptosis and neurological dysfunction after traumatic brain injury. J Neurosci 1997;17: 7415-7424.

86. Feng Y, Fratkin JD, LeBlanc MH. Inhibiting caspase-9 after injury reduces hypoxic ischemic neuronal injury in the cortex in the newborn rat. Neurosci Lett 2003;344:201-204.

87. Fink K, Zhu J, Namura S, et al. Prolonged therapeutic window for ischemic brain damage caused by delayed caspase activation. J Cereb Blood Flow Metab 1998;18:1071-1076.

88. Schulz JB, Weller M, Matthews RT, et al. Extended therapeutic window for caspase inhibition and synergy with MK-801 in the treatment of cerebral histotoxic hypoxia. Cell Death Differ 1998; 5:847-857.

89. Cao G, Xiao M, Sun F, et al. Cloning of a novel Apaf-1-interacting protein: a potent suppressor of apoptosis and ischemic neuronal cell death. J Neurosci 2004;24:6189-6201.

90. Mondragon L, Galluzzi L, Mouhamad S, et al. A chemical inhibitor of Apaf-1 exerts mitochondrioprotective functions and interferes with the intra-S-phase DNA damage checkpoint. Apoptosis 2009;14:182-190.

91. Loetscher H, Niederhauser O, Kemp J, et al. Is caspase-3 inhibition a valid therapeutic strategy in cerebral ischemia? Drug Discov Today 2001;6:671-680.

92. Knoblach SM, Alroy DA, Nikolaeva M, et al. Caspase inhibitor z-DEVD-fmk attenuates calpain and necrotic cell death in vitro and after traumatic brain injury. J Cereb Blood Flow Metab 2004;24:1119-1132.

93. Rami A, Bechmann I, Stehle JH. Exploiting endogenous antiapoptotic proteins for novel therapeutic strategies in cerebral ischemia. Prog Neurobiol 2008;85:273-296.

94. Gill R, Soriano M, Blomgren K, et al. Role of caspase-3 activation in cerebral ischemia-induced neurodegeneration in adult and neonatal brain. J Cereb Blood Flow Metab 2002;22:420-430.

95. Salvesen GS, Duckett CS. IAP proteins: blocking the road to death's door. Nat Rev Mol Cell Biol 2002;3:401-410.

96. Potts PR, Singh S, Knezek M, et al. Critical function of endogenous XIAP in regulating caspase activation during sympathetic neuronal apoptosis. J Cell Biol 2003;163:789-799.

97. Trapp T, Korhonen L, Besselmann M, et al. Transgenic mice overexpressing XIAP in neurons show better outcome after transient cerebral ischemia. Mol Cell Neurosci 2003;23:302-313.
98. Askalan R, Salweski R, Tuor UI, et al. X-linked inhibitor of apoptosis protein expression after ischemic injury in the human and rat developing brain. Pediatr Res 2009;65:21-26.

99. Xu D, Bureau Y, McIntyre DC, et al. Attenuation of ischemiainduced cellular and behavioral deficits by $\mathrm{X}$ chromosome-linked inhibitor of apoptosis protein overexpression in the rat hippocampus. J Neurosci 1999;19:5026-5033.

100. Fan YF, Lu CZ, Xie J, et al. Apoptosis inhibition in ischemic brain by intraperitoneal PTD-BIR3-RING (XIAP). Neurochem Int 2006;48:50-59.

101. Harlin H, Reffey SB, Duckett CS, et al. Characterization of XIAP-deficient mice. Mol Cell Biol 2001;21:3604-3608.

102. Du C, Fang M, Li Y, et al. Smac, a mitochondrial protein that promotes cytochrome c-dependent caspase activation by eliminating IAP inhibition. Cell 2000;102:33-42.

103. Verhagen AM, Ekert PG, Pakusch M, et al. Identification of DIABLO, a mammalian protein that promotes apoptosis by binding to and antagonizing IAP proteins. Cell 2000;102:43-53.

104. Martins LM, Iaccarino I, Tenev T, et al. The serine protease Omi/HtrA2 regulates apoptosis by binding XIAP through a reaperlike motif. J Biol Chem 2002;277:439-444.

105. Gottfried Y, Rotem A, Lotan R, et al. The mitochondrial ARTS protein promotes apoptosis through targeting XIAP. EMBO J 2004;23:1627-1635.

106. Larisch S, Yi Y, Lotan R, et al. A novel mitochondrial septin-like protein, ARTS, mediates apoptosis dependent on its P-loop motif. Nat Cell Biol 2000;2:915-921.

107. Liu Z, Sun C, Olejniczak ET, et al. Structural basis for binding of Smac/DIABLO to the XIAP BIR3 domain. Nature 2000;408: 1004-1008.

108. Yang QH, Church-Hajduk R, Ren J, et al. Omi/HtrA2 catalytic cleavage of inhibitor of apoptosis (IAP) irreversibly inactivates IAPs and facilitates caspase activity in apoptosis. Genes Dev 2003;17:1487-1496.

109. Su D, Su Z, Wang J, et al. UCF-101, a novel Omi/HtrA2 inhibitor, protects against cerebral ischemia/reperfusion injury in rats. Anat Rec (Hoboken) 2009;292:854-861.

110. Kissel H, Georgescu MM, Larisch S, et al. The Sept4 septin locus is required for sperm terminal differentiation in mice. Dev Cell 2005;8:353-364.

111. Russell JC, Whiting H, Szuflita N, et al. Nuclear translocation of $\mathrm{X}$-linked inhibitor of apoptosis (XIAP) determines cell fate after hypoxia ischemia in neonatal brain. J Neurochem 2008;106: 1357-1370.

112. Cao G, Clark RS, Pei W, et al. Translocation of apoptosis-inducing factor in vulnerable neurons after transient cerebral ischemia and in neuronal cultures after oxygen-glucose deprivation. J Cereb Blood Flow Metab 2003;23:1137-1150.

113. Ferrer I, Friguls B, Dalfo E, et al. Caspase-dependent and caspase-independent signalling of apoptosis in the penumbra following middle cerebral artery occlusion in the adult rat. Neuropathol Appl Neurobiol 2003;29:472-481.

114. Slemmer JE, Zhu C, Landshamer S, et al. Causal role of apoptosis-inducing factor for neuronal cell death following traumatic brain injury. Am J Pathol 2008;173:1795-1805. 